\title{
Mudanças na qualidade durante a maturação de frutos de cajazeiras ${ }^{1}$
}

\author{
Edileide Natália da Silva Rodrigues ${ }^{2}$, Silvanda de Melo Silva ${ }^{3}$, Tatiana Leite Bulhões ${ }^{4}$, Raylson de Sá \\ Melo $^{5}$, Alex Sandro Bezerra de Sousa ${ }^{6}$, Renato Pereira Lima ${ }^{7}$, Rejane Maria Nunes Mendonça ${ }^{8}$
}

\begin{abstract}
Resumo: O cajá (Spondias mombin L.) apresenta elevada aceitação devido ao seu sabor exótico. O entendimento do processo de maturação deste fruto é um fator decisivo para se estabelecer padrões de identidade e qualidade, propiciando a previsão da vida útil pós-colheita, além de permitir a utilização adequada dos frutos em estádios de máxima qualidade gustativa. Portanto, este trabalho tem como objetivo avaliar as mudanças na qualidade de frutos de cajazeiras de ocorrência no Brejo Paraibano durante a maturação. Os frutos foram colhidos em cinco estádios de maturação, sendo que cada fruto corresponde a uma repetição, totalizando 80 frutos para as avaliações físicas (coloração da casca, índice de cor da casca, comprimento, diâmetro, massa fresca dos frutos e firmeza) e a polpa de quatro repetições de vinte frutos para as físico-químicas (acidez titulável, sólidos solúveis, relação SS/AT, pH e ácido ascórbico). Os dados foram submetidos à ANOVA e ao teste de Tukey. Frutos dos estádios início de pigmentação (IP), amarelo esverdeado (AV), verde amarelado (VA) e totalmente amarelado (TA) apresentaram maiores massa, comprimento e diâmetro. A transição da cor verde para a amarela ocorreu entre os estádios IP e VA. Frutos do estádio TA se destacaram como mais saborosos, pelos maiores teores de sólidos solúveis $(15,42 \%)$, relação SS/AT $(7,40)$ e significativo aporte de ácido ascórbico $\left(37,85 \mathrm{mg} \cdot 100 \mathrm{~g}^{-1}\right)$, que valoriza o seu consumo.
\end{abstract}

Palavras-chave: Spondias mombin L.; evolução da maturação; coloração; relação SS/AT; ácido ascórbico.

\section{Changes in quality during maturation of yellow mombin fruits}

\begin{abstract}
Yellow mombin fruit is highly accepted because of its exotic taste. The understanding of the maturation process of these fruits is a decisive factor to establish identity and quality standards, providing a prediction of the post-harvest life, besides allowing the appropriate use of the fruits in stages of maximum gustatory quality. Therefore, this work aims to evaluate the changes in the quality of yellow mombin fruit occurring in Brejo Paraibano during maturation. The fruits were harvested in five stages of maturation and were used in 80 repetitions of a fruit for the physical evaluations (peel color, peel color index, length, diameter, fresh mass and firmness) and the pulp of four repetitions of twenty fruits for the physical-chemical (titratable acidity, soluble solids, SS/TA ratio, $\mathrm{pH}$ and ascorbic acid). The data were submitted to ANOVA and the Tukey test. Fruits of the stages start of pigmentation (IP), greenish yellow (AV), yellowish green (VA) and totally yellowish (TA) presented higher mass, length and diameter. The transition from green to yellow occurred between the IP and VA stages. Fruits of stage TA stood out as tastier, by the higher contents of soluble solids $(15.42 \%)$, ratio SS/TA $(7,40)$ and significant contribution of ascorbic acid $(37.85 \mathrm{mg} .100 \mathrm{~g}-1)$, which values its consumption.
\end{abstract}

Keywords: Spondias mombin L.; maturation evolution; coloration; ratio SS/TA; ascorbic acid.

\footnotetext{
${ }^{1}$ Submetido em 17/05/2018 e a provado em 02/11/2018

${ }^{2}$ Graduando em agronomia, Universidade Federal da Paraíba (UFPB), Centro de Ciências Agrárias, Areia-PB, CEP: 58397-000; edileidenatalia@hotmail.com

${ }_{3}^{3}$ Doutora em Horticultura; Professora Titular, Universidade Federal da Paraíba (UFPB), Centro de Ciências Agrárias, Departamento de Química e Física, Areia-PB, CEP: 58397-000; E-mail: silvandasilva@gmail.com

${ }^{4}$ Graduando em agronomia, Universidade Federal da Paraíba (UFPB), Centro de Ciências Agrárias, Areia-PB, CEP: 58397-000; tatianaleitesd@gmail.com

${ }^{5}$ Mestre em Agronomia; Doutorando em Agronomia/Fitotecnia, Universidade Federal do Ceará (UFC), Centro de Ciências Agrárias, Programa de Pós-graduação em Agronomia/Fitotecnia, Fortaleza - CE, CEP: 60440-544; E-mail: raylsonmello@gmail.com

${ }^{6}$ Mestre em Agronomia; Doutorando em Agronomia, Universidade Federal da Paraíba (UFPB), Centro de Ciências Agrárias, Programa de Pósgraduação em Agronomia, Areia-PB, CEP: 58397-000; E-mail: alexsandrosousa.b@gmail.com

${ }_{7}^{7}$ Mestre em Agronomia; Doutorando em Agronomia, Universidade Federal da Paraíba (UFPB), Centro de Ciências Agrárias, Programa de Pósgraduação em Agronomia, Areia-PB, CEP: 58397-000; E-mail: renatolima.p@gmail.com

${ }^{8}$ Doutora em Fitotecnia; Professor associado I, Universidade Federal da Paraíba (UFPB), Centro de Ciências Agrárias, Departamento de Fitotecnia e Ciências ambientais, Areia-PB, CEP: 58397-000; E-mail: rejaneufpb@yahoo.com.br
} 


\section{Introdução}

Spondias é um gênero de árvores frutíferas comestíveis que pertence à família botânica Anacardeaceae, que compreende 18 espécies nativas (Mitchell e Daly, 2015). Os frutos de frutíferas do gênero Spondias são amplamente apreciados pela população do Semiárido para a qual representam emprego e renda, a exemplo do umbuzeiro (Lima et al., 2018), umbugueleira (Dantas et al., 2016); umbu-cajázeira (Gondim et al., 2013) e a cajazeira (Silva et al., 2013). Neste contexto, dentre essas frutíferas, a cajazeira (Spondias mombin L.) é uma planta nativa da América tropical (Tiburski et al., 2011).

No Brasil, frutos da cajazeira (Spondias mombin L.) recebem nomes tais como cajá verdadeiro, cajá-mirim (Moura et al.,2004), taperebá, na região da Amazônia brasileira, cajá, no Nordeste e na maioria das partes do Brasil (Mitchell e Daly, 2015), sendo geralmente colhidos de áreas de ocorrência espontânea e subespontânea, exigindo ações de domesticação pelo elevado porte, mais $10 \mathrm{~m}$ de altura, e de técnicas de propagação (Souza et al., 2010; Véras et al., 2017) para que este fruto possa se estabelecer nos grandes mercados consumidores (Silva et al., 2012).

A qualidade dos frutos é atribuída aos caracteres físicos, que respondem pela aparência, e ao seu valor nutritivo. Para cajás destinados à indústria de sucos, deve-se dar ênfase ao tamanho, a forma e a coloração, que estão relacionados ao conjunto de outros atributos referentes ao sabor, odor, textura alto rendimento em polpa, boa consistência, maior teor de açúcar e acidez elevada (Gondim et al., 2013).

O cajá é uma drupa ovoide, com 3 e $5 \mathrm{~cm}$ de comprimento, que quando totalmente maduro apresenta pele fina de coloração amarelodourado, com polpa suavemente translúcida e suculenta (Morton, 2013), sabor agridoce agradável (Tiburski et al., 2011) e aroma exótico (Neiens et al., 2017), que tem despertado interesse por estudos mais aprofundados devido alegações do seu potencial funcional (Silva et al., 2012). Portanto, este fruto possui alto potencial como matéria prima para a indústria (Silva et al., 2013). Entretanto, a sua industrialização é totalmente dependente da variação sazonal da fruta, levando em consideração a exploração extrativa da árvore de cajazeira e as perdas de frutos elevados devido à práticas precárias de colheita, manuseio pós-colheita e transporte (Silva et al., 2013).

Apesar do aumento da produção e tentativas de domesticação da cajazeira em anos recentes, as iniciativas de implantação de pomares comerciais no Brasil são escassas. Portanto, os frutos no mercado são na grande maioria obtidos durante a exploração extrativista de plantas de ocorrência espontânea, resultado em elevadas perdas póscolheita (Sousa et al., 2010). Entretanto, a sua ampla exploração comercial é dependente da produção racional, estabelecimentos de padrões de identidade e qualidade e de práticas adequadas de colheita e manuseio pós-colheita e transporte que propicie reduzir os níveis elevados as perdas pós-colheita deste fruto (Silva et al., 2013).

Neste contexto, sendo uma frutífera de ocorrência espontânea, os frutos apresentam elevada variabilidade genética. Portanto, estudos que identifique a variabilidade nas espécies são pré-requisitos essenciais para sua preservação e para o sucesso de programas de melhoramento (Lima et al., 2011), No entanto, as informações sobre os aspectos de qualidade deste fruto ainda são pouco consistentes e representativas (Silva et al., 2012).

Aliado a isso, o alto porte e longo período juvenil da cajazeira restringe instalações de experimentos de campos, além de representarem consideráveis obstáculos ao cultivo (Souza et al., 2010; Silva et al., 2013). Portanto, a inserção da cajazeira como espécie frutífera dentro dos modelos agronômicos modernos requer $\mathrm{o}$ desenvolvimento de técnicas adequadas de propagação (Véras et al., 2017), identificação da capacidade produtiva (Bosco et al., 2000) e de características melhoradas de redução de porte de plantas (Lima et al., 2011), além padronizar a qualidade dos seus frutos (Silva et al., 2012; Gondim et al., 2013). Isso justifica a importância de estudos visando descrever a evolução das características de qualidade durante a maturação, tendo em vista a escassez de informações para a valorização e do amplo potencial de exploração comercial destes frutos desta espécie.

Diante do exposto, objetivou-se com o trabalho foi avaliar a evolução da qualidade de frutos de cajazeiras durante a maturação de frutos da cajazeira de ocorrência no Brejo Paraibano. 


\section{Material e Métodos}

\subsection{Material vegetal}

Os frutos da cajazeira (Spondias mombim L.) foram colhidos nas primeiras horas da manhã (7:00 ás 9:00 horas) de 10 plantas no município de Areia-PB (6 $\left.58^{\prime} 22^{\prime \prime} \mathrm{S}, 35^{\circ} 42^{\prime} 57^{\prime \prime}\right)$, Brejo Paraibano, em cinco estádios de maturação, classificados de acordo com a coloração da casca dos frutos: verde (V); início de pigmentação (IP); VA- Verde amarelado; AE- Amarelo esverdeado; TA- Totalmente amarelo (Figura 1.).

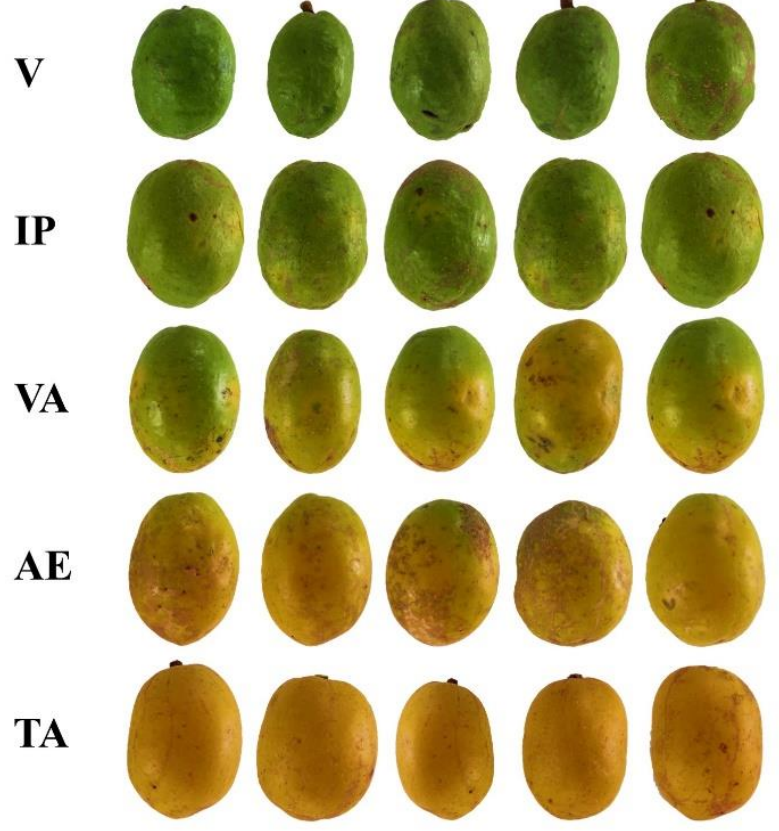

Figura 1 Frutos de cajazeiras (Spondias mombim L.) colhidos com base na coloração da casca, maturação verde (V), início de pigmentação (IP), verde amarelado (VA), amarelo esverdeado (AE) e totalmente amarelado (TA) no município de Areia, estado da Paraíba, Brasil.

Logo após a colheita os frutos foram acondicionados em sacos plásticos e transportados em caixas isotérmicas para $\mathrm{O}$ Laboratório de Biologia e Tecnologia PósColheita do Centro de Ciências Agrárias (CCA) da Universidade Federal da Paraíba (UFPB). As avaliações das características físicas foram utilizados 80 frutos para cada estádio de maturação, sendo cada fruto correspondente a uma repetição. Para as avaliações físicoquímicas, a polpa de 20 frutos foi homogeneizada por repetição, num total de 4 repetições.

\subsection{Avaliações}

As avaliações, realizadas de acordo com Gondin et al. (2013), Silva et al. (2013) e Moura et al. (2004), foram as seguintes:

Coloração da casca: realizada com colorímetro digital Minolta, avaliado pelo sistema CIELAB, correspondente a: $\mathrm{L}^{*}$ - claridade/luminosidade; $a^{*}$-define a transição da cor verde $\left(-a^{*}\right)$ para a cor vermelha $\left(+a^{*}\right) ; b^{*}$ - a transição da cor azul $\left(-b^{*}\right)$ para a cor amarela $\left(+b^{*}\right)$, por refletância. Em cada fruto, realizou-se duas leituras na zona central.

Índice de cor da casca (IC): calculado a partir dos valores de $L, a^{*}$ e $b^{*}$, utilizando equação proposta por Camelo e Gomes (2004), $\mathrm{IC}=2000 *(\mathrm{a} *) / \mathrm{L} *\left(\sqrt{ }\left(\mathrm{a}^{*}\right)+\left(\mathrm{b}^{*}\right)^{2}\right)$.

Comprimento e diâmetro (mm): determinados com paquímetro digital, obtendo as medidas na direção paralela (zona vertical) e perpendicular (zona horizontal) em relação ao eixo central dos frutos íntegros.

Massa fresca dos frutos (g): através de pesagem individual do fruto em balança semi-analítica.

Firmeza (N): determinada com Penetrômetro de bancada Magness Taylor Pressure Tester ${ }^{\circledR}$, sendo realizada a leitura na zona central do fruto.

Acidez titulável (g de ácido cítrico $100 \mathrm{~g}^{-1}$ de polpa): determinada através de titulometria utilizando solução de $\mathrm{NaOH} \quad 0,1 \mathrm{M}$ com indicador fenolftaleína, até obtenção de coloração róseo claro permanente, utilizando 1 $\mathrm{g}$ da amostra em $50 \mathrm{~mL}$ de água destilada conforme metodologia Instituto Adolf Lutz (2008), e calculada pela fórmula: (V x F x M x $\mathrm{PM}) /(10 \times \mathrm{P} \times \mathrm{n})$, onde $\mathrm{V}=$ volume gasto de $\mathrm{NaOH}$ na titulação em $\mathrm{mL} ; \mathrm{F}$ fator de correção da solução de $\mathrm{NaOH} ; \mathrm{M}=$ molaridade da solução de hidróxido de sódio; $\mathrm{PM}=$ peso molecular do ácido correspondente em $\mathrm{g} ; \mathrm{P}=$ massa da amostra em g; e $\mathrm{n}=$ número de hidrogênios ionizáveis do ácido predominante na amostra.

Sólidos solúveis $(\%)$ : determinados por meio de leitura direta com refratômetro de bancada, tipo Abbe digital, ATAGO N1, regulado a $20^{\circ} \mathrm{C}$.

Relação SS/AT: mediante divisão dos índices de SS por AT.

Potencial hidrogeniônico (pH): com potenciômetro digital. 
Ácido ascórbico (AA - mg $\mathbf{1 0 0 g}^{-1}$ ): determinado por titulometria, utilizando-se solução de DFI (2,6 dicloro-fenol -indofenol $0,002 \%$ ) até obtenção de coloração róseo claro permanente, utilizando-se $1 \mathrm{~g}$ da amostra em $50 \mathrm{~mL}$ de Ácido Oxálico 0,5\% conforme Strohecker e Henning (1967), calculado pela fórmula: $(\mathrm{VxF} / 1000)$ $* 100 / \mathrm{P}$, onde $\mathrm{V}=$ volume de DFI gasto na titulação; $\mathrm{F}=$ título do $\mathrm{DFI}$; e $\mathrm{P}=$ peso da amostra.

\subsection{Análise estatística}

Os dados das avaliações foram submetidos à análise de variância (ANOVA), utilizando o software Sisvar, versão 5.6. (Ferreira, 2010). Na comparação entre os estádios de maturação foi utilizado o teste de Tukey em até 5\% de probabilidade de erro.
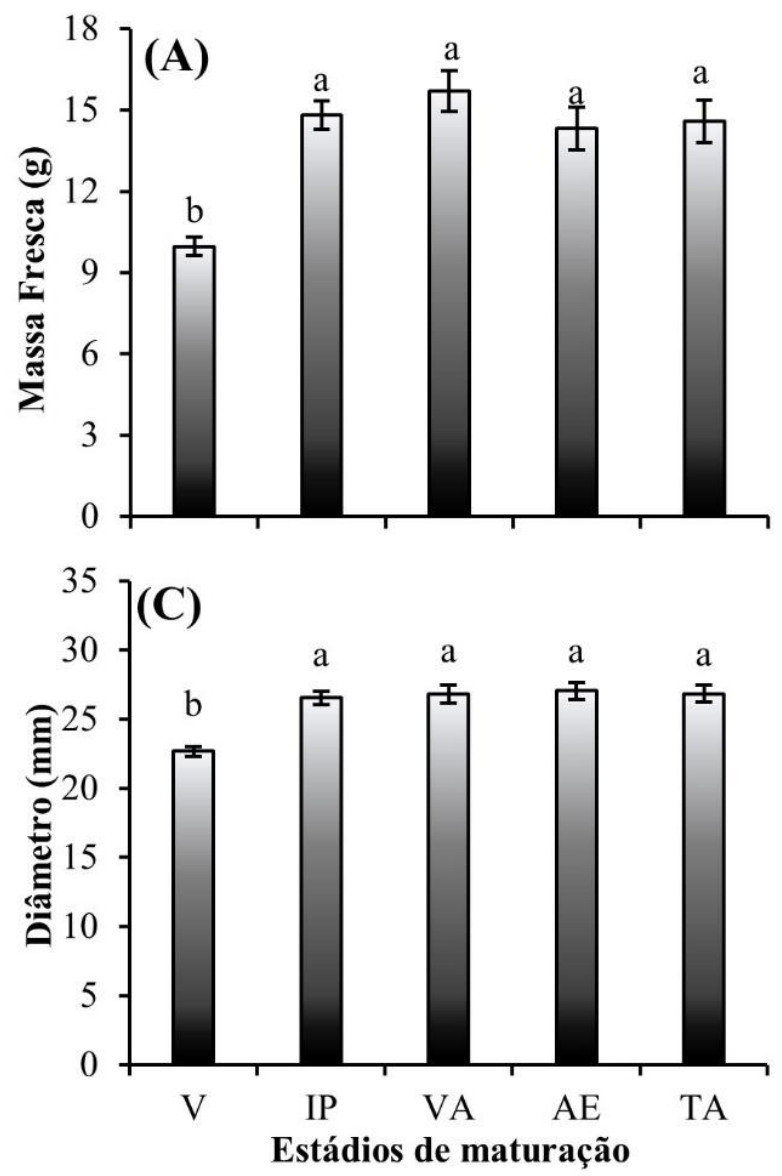

\section{Resultados e Discussão}

\subsection{Características físicas e cor}

A massa fresca foi menor em frutos do estádio verdes $(\mathrm{V})$, com média de 9,96g. Os frutos dos demais estádios apresentaram massas de 14,79 (IP), 15,70 (VA), 14,32 (AE) e 14,57g (TA), não diferindo entre si (Figura 2A). Para Silvino et al. (2017), a massa de cajá variou de 4,75 a $13,73 \mathrm{~g}$, inferiores à deste trabalho, e Carvalho et al. (2011) reportou massas médias variando entre 6,15 a $18,82 \mathrm{~g}$, este último superior aos aqui reportado, indicando a variação da massa de frutos entre regiões de ocorrência.
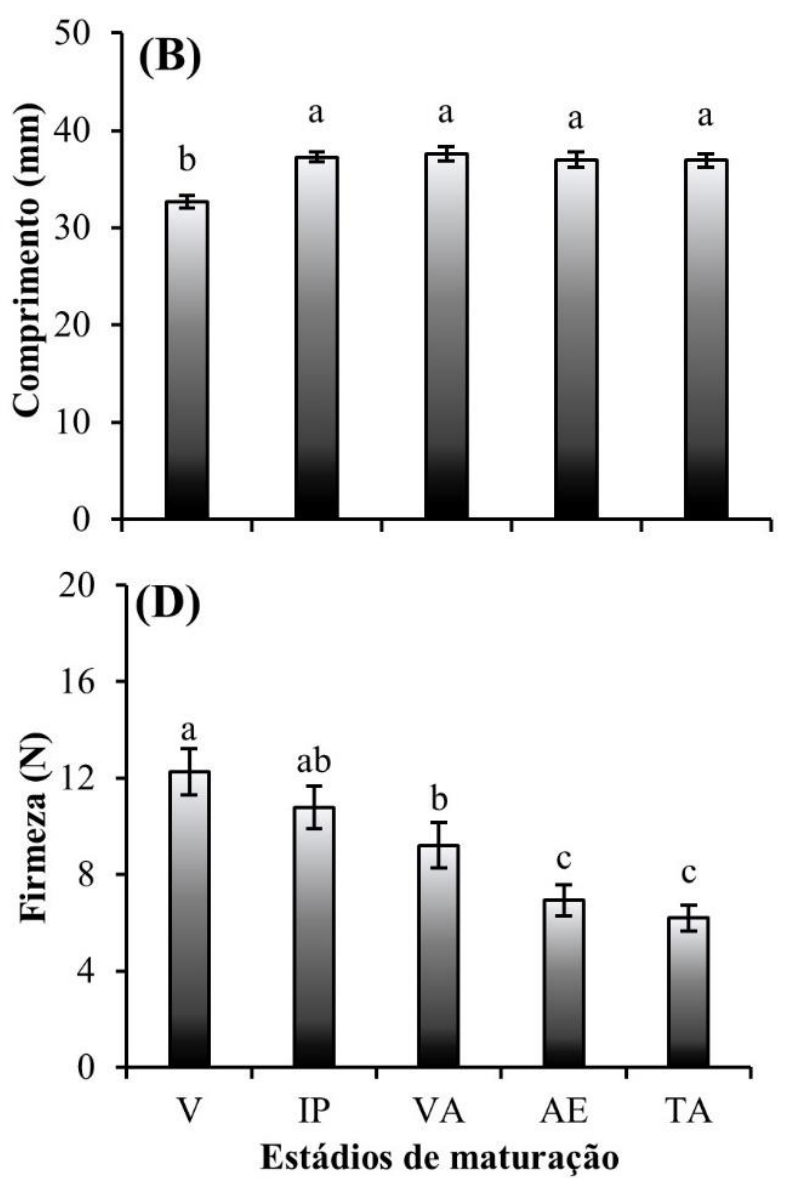

Figura 2 Valores médios e erro padrão da média para a massa fresca (A), comprimento (B), diâmetro (C) e firmeza (D) de frutos de cajazeiras (Spondias mombin L.) colhidos nos estádios de maturação baseada na coloração da casca verde (V), início de pigmentação (IP), verde amarelado (VA), amarelo esverdeado (AE) e totalmente amarelo (TA), de plantas de ocorrência espontânea do Brejo Paraibano. Médias seguidas pela mesma letra não diferem entre si pelo teste de Tukey $(\mathrm{p} \leq 0,05)$. 
De acordo com a classificação de Bosco et al. (2000), cajá com massa superior a 15 g está na classe fruto grande, fruto médio entre $12 \mathrm{e} 15 \mathrm{~g}$ e, pequeno, com massa inferior a $12 \mathrm{~g}$. Portanto, os cajás aqui avaliados podem ser classificados de tamanhos pequeno (estádio V), médio (estádios início de pigmentação (IP), amarelo esverdeado (AV) e totalmente amarelo (TA) e grande (estádio verde amarelado (VA)).

O comprimento de cajá variou de $32,63 \mathrm{~mm}$ (V) a 37,60 mm (VA), no entanto cajás nos três últimos estádios de maturação não diferiram entre si (Figura 2B). Estes valores estão na faixa dos reportados por Bora et al. (1991), que variava entre 35 até $60 \mathrm{~mm}$. Os comprimentos de cajás deste trabalho, no entanto, são superiores os relatados por Carvalho et al. (2011) entre 26,4 a $33,8 \mathrm{~mm}$ e, mais recentemente, por Silvino (2017) entre 21,8 a $35,3 \mathrm{~mm}$.

O diâmetro dos frutos variou de 22,67 (V) a $27,06 \mathrm{~mm}$ (AV) (Figura 2C). Portanto os frutos do estádio de maturação verde apresentaram o menor tamanho. Silvino et al. (2017) reportou diâmetro variando entre 18,3 a 28,1 mm e Soares et al. (2006) entre 18,3 a 26,8 mm, ambos na faixa dos deste trabalho. Estes diâmetros, entretanto, são inferiores aos encontrados por Santos (1996), entre 29,11 a $29,57 \mathrm{~mm}$.

De acordo com Chitarra e Chitarra (2005) quando o quociente entre o comprimento e o diâmetro se aproximar de 1.0, o fruto terá formato mais arredondado. Portanto os frutos mais maduros (estádio amarelo esverdeado e totalmente amarelado) são mais arredondados, pois seus quocientes entre comprimento e diâmetro foram os que mais se aproximaram de 1 .

A firmeza dos frutos de cajazeiras diminuiu com o avanço da maturação, de 12,26 $\mathrm{N}$ em frutos verdes (V) para 6,20 N nos totalmente amarelados (TA), correspondente a um declínio de 50,57\% entre frutos destes estádios de maturação (Figura 2D). A perda da firmeza durante a maturação está associada à degradação de polímeros da parede celular, a exemplo de celulose, hemicelulose, pectinas, ligninas entre outros, resultado em perda da coesão entre as células e na perda de resistência dos tecidos (Chitarra e Chitarra, 2005).

A luminosidade (L) dos frutos aumentou com o avanço da maturação (Figura 3A). O parâmetro $a^{*}$ (Figura 3B) migrou de um valor negativo (coloração verde) para positivo (amarela), na transição do estádio IP para o VA. O parâmetro $b^{*}$ aumentou com o avanço da maturação, com maior valor em frutos no estádio VA, com uma pequena diminuição em seguida em frutos TA (Figura 3C). Em frutos verdes o valor médio de $b^{*}$ foi 30,51; para os verdes amarelados foi 48,28 e em frutos totalmente amarelos a média foi 46,96 . Maldonado Astudillo et al. (2014) observaram aumento nos valores de parâmetros de cor de ciriguelas armazenadas no estádio semi-madura, corroborando com os resultados encontrados aqui neste trabalho.

O índice de cor da casca indicou claramente que a transição da coloração verde para o amarelo durante a maturação de cajá ocorre na mudança da pigmentação amarela (IP) para o verde amarelado (VA), de modo que a degradação da clorofila dar lugar à definição da coloração amarela (Figura 3D). Comparando o índice de cor da casca com as variáveis estudadas, observou-se que a firmeza do cajá declina a medida que coloração amarela evolui durante a maturação, de modo que a mudança mais evidente ocorre no estádio VA. Portanto, pode-se inferir que o estádio VA se caracteriza como uma interface para o amadurecimento de cajá. Rodrigues et al. (2018) também reportou correlação do declínio da firmeza com a evolução da coloração amarela em de goiaba 'Paluma' recoberta com amido de jaca e quitosana.

Durante o amadurecimento, a cor da casca do cajá evoluiu do verde ao amarelo, devido à degradação gradual da clorofila pela ação enzimática, o que permite aos carotenoides tornarem-se evidentes (Heaton \& Marangoni, 1996; Matile et al., 1999; Matsuura, et al., 2002; Rodrigues et al., 2018), principalmente pela ação da clorofilase nos cloroplastos (Chitarra e Chitarra, 2005). Li et al. (1997) observaram que altos teores de clorofila implicam em menores valores de $\mathrm{L}^{*}, a^{*}, b^{*}$, sendo que este último parâmetro representa o comprimento de onda da cor dominante, podendo prever os conteúdos das clorofilas a e b, com altos valores nos coeficientes de correlação. A coloração um importante atributo de qualidade dos frutos frescos, pois é uma característica que atrai o consumidor no primeiro momento (Moura et al., 2004). 

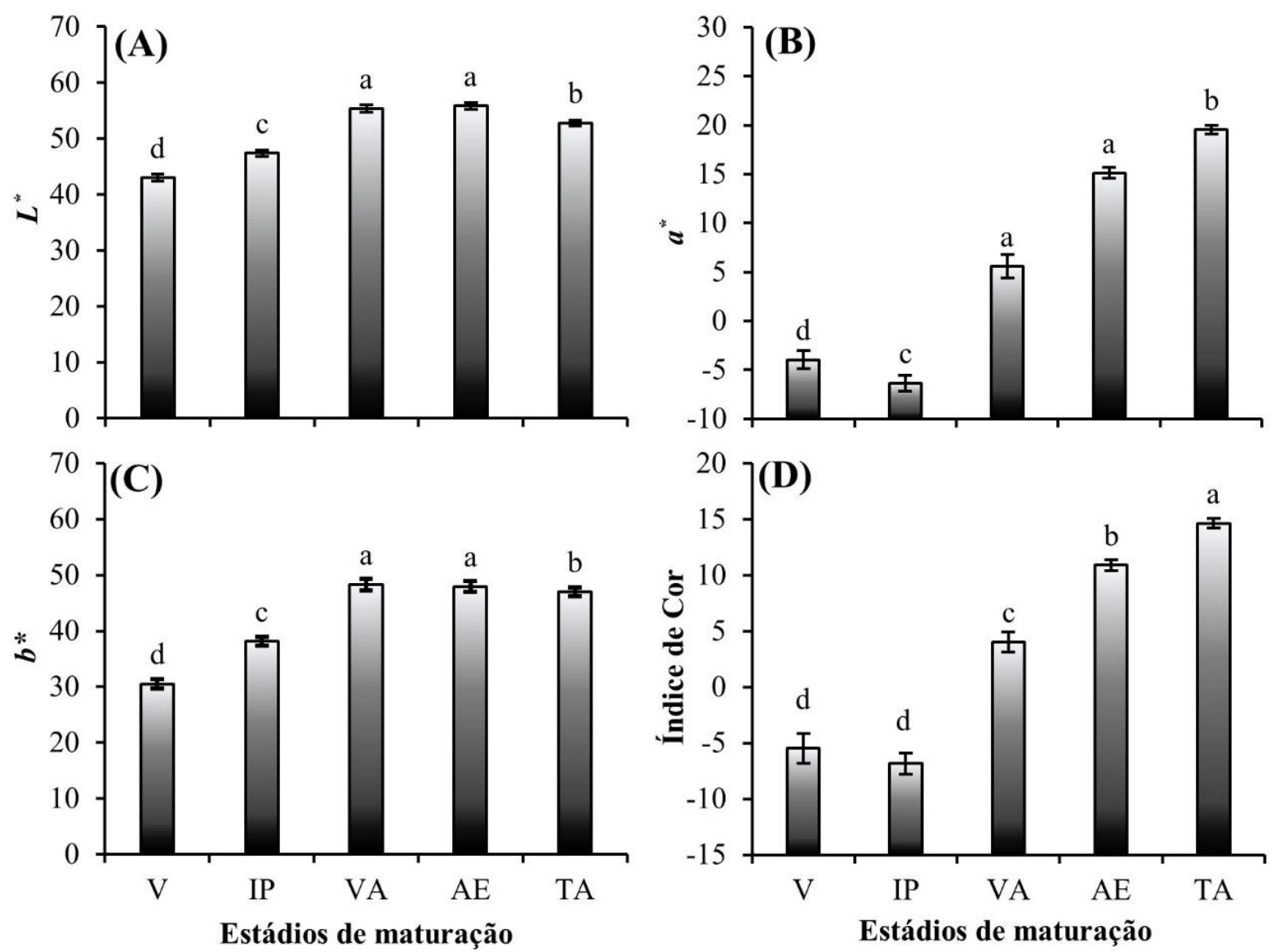

Figura 3 Valores médios e erro padrão da média para evolução da coloração, expressa através de (A) luminosidade (L), (B) $a^{*}$ (transição da cor verde (-a*) para a cor vermelha $\left(+\mathrm{a}^{*}\right)$ e (C) $b^{*}$ (transição da cor azul (-b*) para a cor amarela $\left(+b^{*}\right)$, e (D) índice de coloração, em frutos de cajazeiras (Spondias mombim L.) colhidos nos estádios de maturação baseada na coloração da casca verde (V), início de pigmentação (IP), verde amarelado (VA), amarelo esverdeado (AE) e totalmente amarelo (TA), de plantas de ocorrência espontânea do Brejo Paraibano. Médias seguidas pela mesma letra não diferem entre si pelo teste de Tukey $(p \leq 0,05)$.

\subsection{Características da polpa}

O conteúdo de sólidos solúveis (SS) em cajá aumentou durante a maturação, variando de $8,25 \%$ no estádio V, para $15,42 \%$ no TA (Figura 4A). Frutos do estádio V apresentaram teor de SS abaixo do mínimo exigido $(9,00 \%)$ pelo Padrão de Identidade e Qualidade (PQI) para polpa de cajá (Brasil, 1999), como também reportado para umbu-cajá por Gondim et al. (2013), todos em estádio de maturação totalmente amarelado. Silvino et al. (2017) em matéria seca de cajá reportaram SS médio de 10,00\% obtido de frutos totalmente amarelados secos em estufa, próximo ao de Mattietto et al. (2010) avaliado em frutos totalmente amarelados.

De acordo com Chitarra e Chitarra (2005) o teor de SS tende a aumentar durante a maturação devido a biossíntese de açúcares solúveis ou a degradação de polissacarídeos. Este teor é frequentemente usado como um indicador de amadurecimento e de sabor da frutas e quanto menor os SS, mais ácida e / ou menos doce será a polpa ou suco (Hui \& Barta, 2006).

A acidez titulável (AT) do cajá não diferiu com o avanço da maturação (Figura 4B). Entretanto, frutos verdes apresentaram maior AT

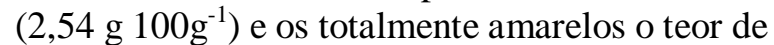
AT atingiu 2,08 g $100 \mathrm{~g}^{-1}$ de ácido cítrico, caracterizando frutos de menor acidez, como também reportado para umbu-cajá por Gondim et al. (2013). O teor de AT deste trabalho foi superior ao de Silvino et al. (2017) avaliando matéria seca de cajá com g $100 \mathrm{~g}^{-1}$ e Carvalho et al. (2011) em cajás colhidos totalmente amarelados, com teores entre 1,20 a $1,93{\mathrm{~g} 100 \mathrm{~g}^{-}}^{-}$ 1. 

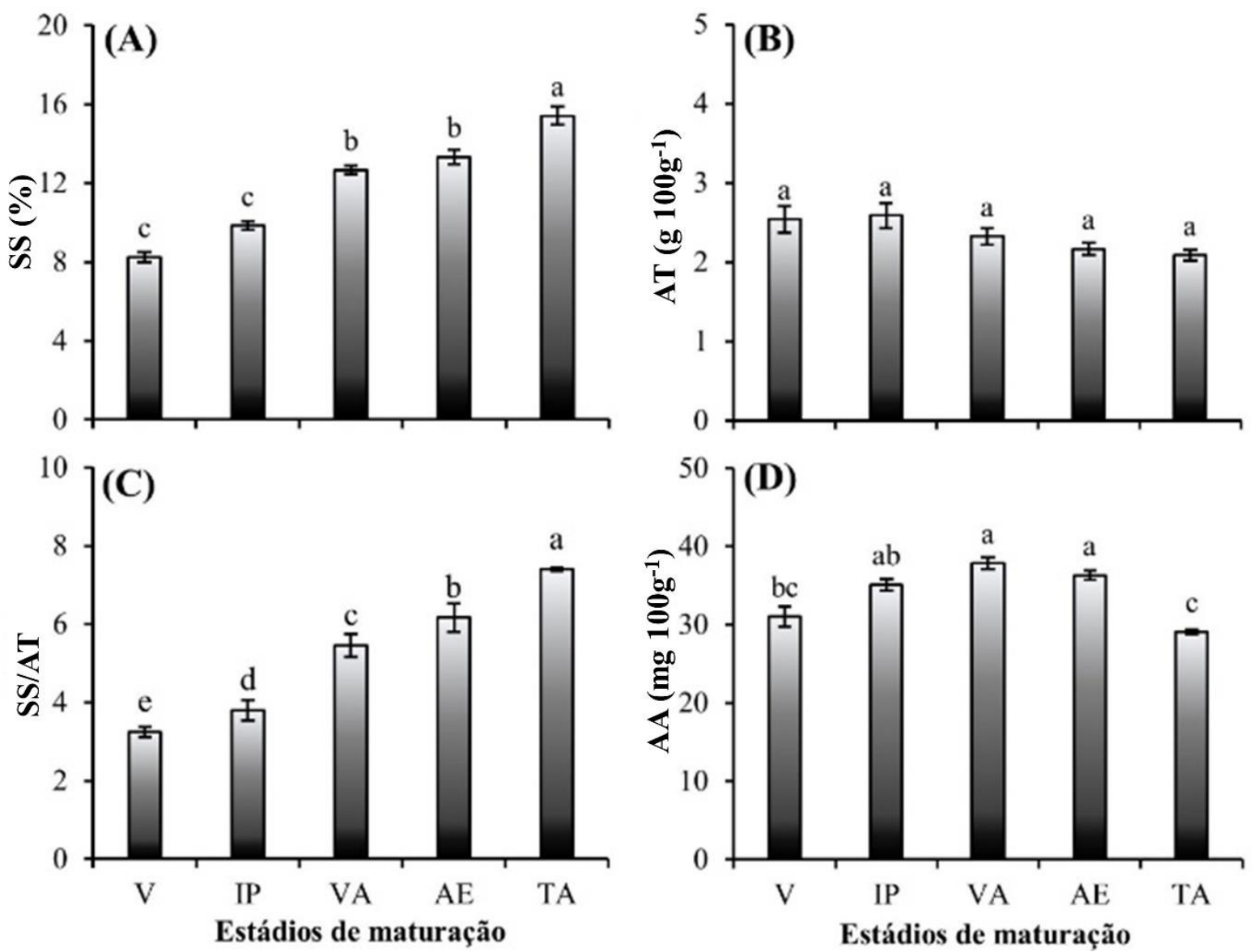

Figura 4 Valores médios e erro padrão da média para sólidos solúveis (SS), acidez titulável (TA), relação SS/AT e ácido ascórbico (AA - mg $100 \mathrm{~g}^{-1}$ ) de frutos de cajazeiras (Spondias mombin L.) colhidos nos estádios de maturação baseada na coloração da casca verde (V), início de pigmentação (IP), verde amarelado (VA), amarelo esverdeado (AE) e totalmente amarelo (TA), de plantas de ocorrência espontânea do Brejo Paraibano. Médias seguidas pela mesma letra não diferem entre si pelo teste de Tukey $(\mathrm{p} \leq 0,05)$.

De acordo com Filgueiras et al. (2001), embora o cajá seja um fruto aromático, seu teor de açúcar é baixo e este apresenta maior acidez quando comparada com outros fruto tropicais, como maracujás e abacaxi. O teor de ácidos orgânicos, geralmente diminui com a maturação dos frutos, em decorrência do processo respiratório ou de sua conversão em açúcares (Chitarra e Chitarra, 2005).

A relação SS/AT aumentou durante a maturação (Figura 4C), atingindo valor máximo $(7,40)$ em frutos totalmente amarelados (TA). López et al. (2004) ao avaliar o efeito do grau de maturidade na qualidade pós-colheita de ciriguelas, encontrou valores para essa relação SS/AT de 23,4 para frutos maduros, sendo este superior ao encontrado neste estudo. A relação SS/AT é um indicativo grau de maturação e, portanto, do sabor da maioria dos frutos em relação ao equilíbrio doce/ácido do fruto (Chitarra e Chitarra, 2005). Assim, esta relação expressa a palatabilidade do fruto e quanto mais alta mais saboroso é o fruto (Dantas et al., 2016).

Os frutos da cajazeira apresentam conteúdo apreciável de ácido ascórbico que aumenta com a evolução da maturação, até o estádio VA, declinando em seguida. Os teores de ácido ascórbico diferiram entre estádios de maturação (Figura 4D). Frutos verdes amarelados e amarelos esverdeados apresentaram os maiores teores de ácido ascórbico com médias de 37,85 e 36,31 mg $100 \mathrm{~g}^{-1}$, respectivamente, seguidos dos em início

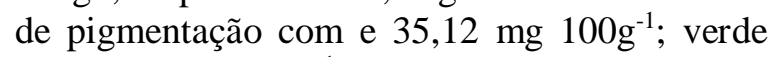

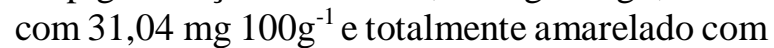
$29,10 \mathrm{mg} 100 \mathrm{~g}^{-1}$. O aporte de ácido ascórbico de cajá contribui para o significativo potencial 
antioxidante alegado a este fruto por Silva et al. (2013) e, portanto, para a valorização do consumo de sua polpa. Os conteúdos de ácido ascórbico deste estudo foram superiores aos reportados por Silvino et al. (2017) na matéria seca de frutos totalmente amarelado, em média de $25,37 \mathrm{mg}$ $100 \mathrm{~g}^{-1}$ cajá, mas foram próximos de Mattieto et al. (2010), com médias de 23,72 mg $100 \mathrm{~g}^{-1}$ em frutos totalmente amarelados.

\section{Conclusão}

Para cajazeiras de ocorrência no Brejo Paraibano com base na evolução da biometria, coloração, firmeza e características físicoquímicas, o ponto de colheita é atingido na planta quando fruto atinge o início da pigmentação amarela, assim, garantindo a evolução da maturação e amplo uso para a indústria e melhor aptidão para o mercado de frutos frescos;

Na evolução da maturação do cajá a transição para o amadurecimento ocorreu entre os estádios início de pigmentação amarela (IP) e verde amarelado (VA), com base principalmente, no índice de cor e perda da firmeza dos frutos. Cajás do estádio totalmente amarelado (TA) se destacaram por apresentar tonalidade amarelolaranja com elevado brilho e intensidade. Além disso, frutos do estádio TA mostraram-se com maior equilíbrio entre doçura e acidez pela maior relação SS/AT;

O cajá apresenta significativo aporte de ácido ascórbico que evolui até o intermédio da maturação, que valoriza este fruto para consumo na forma de polpa e produtos derivados.

\section{Agradecimentos}

Os autores agradecem ao Conselho Nacional de Desenvolvimento Científico e Tecnológico CNPq pelo apoio financeiro.

\section{Referências}

Bora, P. S.; Narain, N.; Holschuh, H. J.; Vasconcelos, M. A. S. Changes in Physical and Chemical Composition during Maturation of Yellow Mombin (Spondias mombin) Fruits. Food Chemistry, v.41, n.3, p.341-348, 1991. https://doi.org/10.1016/0308-8146(91)90058-V

Bosco, J.; Soares, K.T.; Aguiar Filho, S.P.; Barros, R.V. A cultura da cajazeira. João Pessoa: EMEPA, 2000. 29p.
BRASIL. Ministério da Agricultura e do Abastecimento. Instrução Normativa ${ }^{\circ} 122$, de 10 de setembro de 1999. Diário Oficial da República Federativa do Brasil, Brasília, 13 de set. de 1999. Seção 1, p. 72-76.

Camelo, A. F. L.; Gomes, P. A. Comparison of color indexes for tomato ripening. Horticultura Brasileira, v.22, n.3, p.534-537, 2004. http://dx.doi.org/10.1590/S0102-05362004000300006

Carvalho, A. V.; Cavalcante, M. A.; Santana, C. L. Alves, R. M. Características físicas, químicas e atividade antioxidante de frutos de matrizes de cajazeira no estado do Pará. Alimentos e Nutrição Araraquara, v.22, n.1, p.45-53. 2011. http://ainfo.cnptia.embrapa.br/digital/bitstream/item/5666 3/1/1067.pdf

Dantas, A. L; Silva, S. M.; Dantas, R. L.; Sousa, A. S. B.; Schunemann, Pereira, A. P. Desenvolvimento, fisiologia da maturação $\mathrm{e}$ indicadores do ponto de colheita de frutos da umbugueleira (Spondias sp.). Revista Brasileira de Fruticultura, v.38, n.1, p.33-42, 2016. http://dx.doi.org/10.1590/0100-2945-271/14

Ferreira, D. F. Sisvar: Sistema de análise de variância. Versão 5.6. Lavras: UFLA/DEX, 2010.

Filgueiras, H. A. C.; Alves, R. E.; Oliveira, A. C.; Moura, C. F. H.; Araújo, N. C. C. Calidad de Frutas Nativas de Latinoamericana para Industria: Jobo (Spondia mombin L.). Proc Interam Soc Trop Horticulture, v.43, p.7276, 2001.

Gondim, P. J. S.; Silva, S. De M.; Pereira, W. E.; Dantas, A. L.; Chaves neto, J. R Dos.; Qualidade de frutos de acessos de umbucajazeira (Spondias sp.). Revista Brasileira de Engenharia Agrícola e Ambiental, v.17, n.11 p.217-1221, 2013. http://dx.doi.org/10.1590/S1415$\underline{43662013001100013}$

Heaton, J. W.; Marangoni, A. G.; Chlorophyll degradation in processed foods and senescent plant tissues. Trends Food Science \& $\begin{array}{lllll}\text { Technology, } & \text { v.7, } & \text { n.1, } & \text { p.8-15, } 1996 .\end{array}$ https://doi.org/10.1016/0924-2244(96)81352-5

Hui ,Y. H.; Barta, J. Handbook of fruits and fruit processing. Blackwell, Oxford, 2006. 697p. https://doi.org/10.1002/9780470277737

Li, M.; Slaughter, D. C.; Thompson, J. F. Optical chlorophyll sensing system for banana ripening. 
Postharvest Biology and Technology, Amsterdan, v.12, n.3, p.273-283, 1997. https://doi.org/10.1016/S0925-5214(97)00059-8

Lima, M. A. C.; Silva, S. M. ; Oliveira, V. R.. Umbu (Spondias tuberosa). In: Rodrigues, S.; Silva, E.O.; Brito, E.S. (Org.). Exotic Fruits Reference Guide. $1^{\mathrm{a}}$ ed. London: Academic Press - Elsevier, 2018, v. 1, p. 1-490.

Lima, A. T. B.; Souza, V. A. B.; Gomes, R. L. F.; Lima, P. S. C. Molecular characterization of cajá, Spondias mombin (Anacardiaceae), by RAPD markers. Genetics and Molecular Research, v.10, n.4 , p.2893-2904, 2011. http://www.doi.org/10.4238/2011.November.25.1

López, A. P.; Veloz, C. S.; Galarza, M. L. A.; Lúa, A. M. Efecto del grado de madurez en la calidad y vida postcosecha de ciruela mexicana (Spondias purpurea L.). Revista Fitotecnia Mexicana, v.27, n.2, p.133-139, 2004. https://www.academia.edu/attachments/40376595/downlo ad_file?st=MTU1NDA4NzY4OSwxNzcuODYuMTgwLj Qy\&s=swp-splash-header

Maldonado-Astudillo, Y. I.; Alia-Tejacal, I.; Núñez-Colín, C. A.; Jiménez-Hernández, J.; Pelayo-Zaldívar, C.; López-Martínez, V.; Andrade-Rodríguez, M.; Bautista-Baños, S.; Valle-Guadarrama, S. Postharvest physiology and technology of Spondias purpurea L. and $S$. mombin L. Scientia Horticulturae, v.174, n.22, p.193-206, 2014. https://doi.org/10.1016/j.scienta.2014.05.016

Matile, P.; Hortensteiner, S.; Thomas, H.; Chlorophyll degradation. Annual Review of Plant Physiology and Plant Molecular $\begin{array}{lllll}\text { Biology, v.50, n.1, p.67-95, } 1999 . & \text {. }\end{array}$ https://doi.org/10.1146/annurev.arplant.50.1.67

Mattietto, R. A.; Lopes, A. S.; Menezes, H. C.; Caracterização física e físico-química dos frutos da cajazeira (Spondias mombin L.) e de suas polpas obtidas por dois tipos de extrator. Brazilian Journal of Food Technology, v.13, n.3, p.156-164, 2010. https://doi.org/10.4260/BJFT2010130300021

Melo, R. S. ; Silva, S. M. ; Sousa, A. S. B.; Lima, R. P. ; Dantas, A. L. ; Dantas, R. L. ; Figueiredo, V. M. A. . Maturação e qualidade de frutos de mandacaru (Cereus jamacaru P.DC.) de diferentes bioclimas do estado da Paraíba. Revista Agropecuária Técnica, v.38, n.3, p.160-168,

2017.
Mitchell, J. D.; Daly, D. C. A revision of Spondias L. (Anacardiaceae) in the Neotropics. PhytoKeys v.55, p.1-92, 2015. https://doi.org/10.3897/phytokeys.55.8489

Morton, J. F. Fruits of warm climates. Echo Point Books \& Media, Brattleboro, 2013. 550p.

Moura, F. T.; Silva, S. DE M.; Martins, L. P.; Mendonça, R. M. N.; Alves, R. E.; Filgueiras, H. A. C. Evolução do crescimento e da maturação de frutos de cajazeira (Spondias mombin L). Proceedings of the Interamerican Society for Tropical Horticulture Isth, Miami, v.47, n.1, p.231-233, 2004. http://www.ceinfo.cnpat.embrapa.br/arquivos/artigo_155 7.pdf

Neiens, S. D., Geiblitz, S. M., Steinhaus, M.; Aroma-active compounds in Spondias mombin L. fruit pulp. European Food Research and Technology, v. 243, n.6, p.1073-1081, 2017. https://doi.org/10.1007/s00217-016-2825-7

Rodrigues, A. A. M.; Dantas, A.L.; Silva, A. F.; Santos, L. S.; Moreira, D.N.;; Silva, S.M. Physiology and postharvest conservation of 'Paluma' guava under coatings using jack fruit seed-based starch. Revista Brasileira de Fruticultura, v.40, n.2, p.344-352, 2018. http://dx.doi.org/10.1590/0100-29452018352

Silva, F. V. G da; Silva, S. D. E. M.; Silva, G. C.; Mendonça, R. M. N.; Alves, R. E.; Dantas, A. L. Bioactive compounds and antioxidant activity in fruits of clone and ungrafted genotypes of yellow mombin tree. Ciência e Tecnologia de Alimentos, v.32, n.4, p.685-691, 2012. $\underline{20612012005000101}$

http://dx.doi.org/10.1590/S0101-

Silva, F. V. G.; Silva, S. M.; Silva, G. C.; Schunemann, A. P. P. Quality and antioxidant activity during ripening of fruits from yellow mombin (Spondias mombin L.) Genotypes. Acta Horticulturae, v.1012, n.7, p.843-848, 2013.

http://dx.doi.org/10.17660/ActaHortic.2013.1012.114

Silvino, R. C. A. S.; Silva, G. C. T.; Dos Santos, O. V. Qualidade nutricional e parâmetros morfológicos do fruto cajá (Spondias mombin). Revista Desafios, v.4, n.2, p.1-11, 2017. http://dx.doi.org/10.20873/uft.2359-3652.2017v4n2p3

Souza, E. P.; Mendonça, R. M. N.; Silva, S. M.; Estrela, M. A. ; Souza, A. P.; Silva, G. C. . Enxertia da cajazeira. Revista Brasileira de https://doi.org/10.25066/agrotec.v38i3.33818 
Fruticultura, v.32, n.1, p.316-320, 2010. http://dx.doi.org/10.1590/S0100-29452010005000002

Tiburski, J. H.; Rosenthal, A.; Deliza, R.; Godoy, R. O.; PACHECO, S. Nutritional properties of yellow mombin (Spondias mombin L.) pulp. Food Research International, v.44, n.7, 23262331 , 2011.
Véras, M. L. M.; Mendonça, R. M. N.; Ramires, C. M. C. ; Silva, S. M.; Pereira, W. E. Effect of ethephon and indolebutyric acid on yellow mombin propagation via cutting1. Pesquisa Agropecuaria Tropical, v.47, n.4, p.416-423, 2017. http://dx.doi.org/10.1590/1983-

https://doi.org/10.1016/j.foodres.2011.03.037 